\title{
'Obligate' anaerobic Salmonella strain YB1 suppresses liver tumor growth and metastasis in nude mice
}

\author{
CHANG-XIAN LI ${ }^{1 *}$, BIN YU $^{2,3 *}$, LEI SHI ${ }^{2}$, WEI GENG ${ }^{1}$, QIU-BIN LIN ${ }^{2}$, CHANG-CHUN LING ${ }^{1}$, \\ MEI YANG ${ }^{2}$, KEVIN T. P. NG ${ }^{1}$, JIAN-DONG HUANG ${ }^{2,4}$ and KWAN MAN ${ }^{1}$
}

\author{
${ }^{1}$ Department of Surgery and Centre for Cancer Research; ${ }^{2}$ Department of Biochemistry and Shenzhen Institute of \\ Research and Innovation, University of Hong Kong, Hong Kong 999077, SAR; ${ }^{3}$ Key Laboratory of Optoelectronic \\ Devices and Systems of Ministry of Education and Guangdong College of Optoelectronic Engineering, \\ Shenzhen University, Shenzhen, Guangdong 518060; ${ }^{4}$ Centre for Synthetic Biology Engineering Research, \\ Shenzhen Institutes of Advanced Technology, Shenzhen, Guangdong 518055, P.R. China
}

Received December 17, 2014; Accepted February 4, 2016

DOI: 10.3892/ol.2016.5453

\begin{abstract}
The antitumor properties of bacteria have been demonstrated over the past decades. However, the efficacy is limited and unclear. Furthermore, systemic infection remains a serious concern in bacteria treatment. In this study, the effect of YB1, a rationally designed 'obligate' anaerobic Salmonella typhimurium strain, on liver tumor growth and metastasis in a nude mouse orthotopic liver tumor model was investigated. The orthotopic liver tumor model was established in nude mice using the hepatocellular carcinoma cell line MHCC-97L. Two weeks after orthotopic liver tumor implantation, YB1, SL7207 and saline were respectively administered through the tail vein of the mice. Longitudinal monitoring of tumor growth and metastasis was performed using Xenogen IVIS, and direct measurements of tumor volume were taken 3 weeks after treatment. In vitro, MHCC-97L and PLC cells were incubated with YB1 or SL7207 under anaerobic conditions. YB1 was observed to invade tumor cells and induce tumor cell apoptosis and death. The results revealed that all mice in the YB1 group were alive 3 weeks after YB1 injection while all mice
\end{abstract}

Correspondence to: Professor Kwan Man, Department of Surgery and Centre for Cancer Research, University of Hong Kong, Faculty of Medicine Building, 21 Sassoon Road, Hong Kong 999077, SAR, P.R. China

E-mail:kwanman@hku.hk

Professor Jian-Dong Huang, Department of Biochemistry and Shenzhen Institute of Research and Innovation, University of Hong Kong, Faculty of Medicine Building, 21 Sassoon Road, Hong Kong 999077, SAR, P.R. China

E-mail: jdhuang@hku.hk

*Contributed equally

Key words: YB1, hepatocellular carcinoma, tumor growth, metastasis, apoptosis, immune response in the SL7207 group died within 11 days of the SL7207 injection. The body weight decreased by $\sim 9 \%$ on day 1 after YB1 injection and but subsequently recovered. Liver tumor growth and metastases were significantly inhibited following YB1 treatment. By contrast to the control group, a large number of Gr1-positive cells were detected on days 1 to 21 following YB1 treatment. Furthermore, YB1 also effectively invaded tumor cells and induced tumor cell apoptosis and death. In conclusion, YB1 suppressed liver tumor growth and metastasis in a nude mice liver tumor model. The potential mechanism may be through enhancing innate immune response and inducing tumor cell apoptosis and cell death.

\section{Introduction}

Hepatocellular carcinoma (HCC) is one of the most malignant tumors worldwide (1). Surgical therapies including hepatectomy and liver transplantation are first-line treatments for HCC patients, but the unlikelihood of early diagnosis and the high incidence of tumor recurrence and metastasis following surgery remain major obstacles $(2,3)$. Non-surgical treatments including radiofrequency ablation, alcohol injection and transcatheter arterial chemoembolization have made great advances in recent years. However, they have substantial limitations, including toxicity, insufficient tissue penetration and poor tumor targeting, which together often result in incomplete destruction of the tumors (4-6). Therefore, the development of novel adjuvant therapies for the suppression of liver cancer growth and metastasis is essential.

Researchers are now turning to bacterial treatments and consider this a promising new strategy in cancer therapy. Certain bacterial species including Salmonella, Clostridia and Bifidobacteria have been observed to preferentially replicate and accumulate in tumors (7-11). Through observations and research over the past century, bacteria has been noted to affect the tumor in the following ways $(12,13)$ : i) as a tumoricidal agent; ii) as a vector for gene therapy; iii) as toxins for cancer treatment; iv) as bacterial spores; and v) as an 
immunotherapeutic agent. However, major problems with using bacteria as anti-cancer agents include the systemic infection of bacteria $(7,14)$ and the inability to completely eradicate cancer cells. Wild-type bacteria including Salmonella may induce severe infection, which may result in septic shock and high lethality (7). Therefore, researchers use autotrophic mutations to attenuate the virulence of Salmonella in diverse ways (15).

Previously, we engineered YB1, which was derived from the attenuated Salmonella strain SL7207 (16-21). Although SL7207 has aroA and other pathogenic gene mutations, it is lethal for nude mice if administrated intravenously. In YB1, the essential gene asd was engineered to be controlled by the hypoxic promoter Pept and the antisense aerobic promoter SodA. The asd gene is a key enzyme in the synthesis of diaminopimelic acid (DAP), which is an essential component for the gram-negative bacteria cell wall. A deficiency of asd expression or shortage of extrinsic DAP supply in the environment leads to bacterial lysis in a short period of time. Therefore, without additional DAP, YB1 only survives under hypoxic conditions ( $<0.5 \%$ oxygen) (20). However, the normal functions of YB1 are not affected. In a nude mouse breast tumor model, YB1 was rapidly eliminated from normal organs due to their normoxia conditions. However, in tumors, YB1 accumulated inside the hypoxic region and caused tumor regression (20).

In this study, we explored the potential anti-cancer effect of YB1 on HCC using an orthotopic liver tumor animal model with distant metastatic potential. We observed that liver tumor growth was significantly inhibited following YB1 treatment. Furthermore, YB1 also significantly decreased the incidence of lung metastasis. These results indicate that YB1 has a great potential for liver cancer therapy and it provides a new model to study the mechanisms underlying the high efficacy of bacterial suppression of liver cancer growth and metastasis.

\section{Materials and methods}

Orthotopic nude mice liver tumor model. The orthotopic liver tumor model was established in nude mice (male, 4-6 weeks old, 18-24 g) obtained from the Lab Animal Unit of the University of Hong Kong (22). MHCC-97L cells (6x105; Liver Cancer Institute, Fudan University, China) in $0.1 \mathrm{ml}$ culture medium were injected subcutaneously into the right flank of the nude mice. Once the subcutaneous tumors reached 0.8 to $1 \mathrm{~cm}$ in diameter, they were removed and cut into cubes 1 to $2 \mathrm{~mm}^{3}$ in size, which were subsequently implanted into the left liver lobes of another group of nude mice. Mice were housed at room temperature $\left(20-25^{\circ} \mathrm{C}\right)$ in a standard animal laboratory with free activity and access to water and food. The mice were maintained under constant environmental conditions with a 12-h light-dark cycle. All operations were performed in clean conditions. The present study was licensed according to Animal Ordinance Chapter 340 by the Department of Health, Hong Kong Special Administrative Region (ref. 13-501 in DH/HA\&P/8/2/3 Pt. 52).

Treatment regimen, imaging analysis and sample collection. Two weeks after tumor implantation, a single dose of SL7207 (5x107 CFU; lab stock; Stanford University School of Medicine, Stanford, CA, USA), YB1 $\left(5 \times 10^{7} \mathrm{CFU}\right.$; modified from SL7207) (20) or saline (control group; Thai Otsuka.
Pharmaceutical Co., Ltd., Bangkok, Thailand) was injected via the tail vein. Five mice each from the SL7207 and YB1 groups were used to observe the effect of bacteria on mouse survival. The control group mice $(n=9)$ and the remaining nude mice from the YB1 group $(n=14)$ were used to monitor liver tumor growth and metastasis using a Xenogen in vivo imaging system (PerkinElmer, Inc., Waltham, MA, USA) through the detection of luminance signals from tumor cells. The mice were sacrificed 3 weeks after the YB1 or saline injection. The tumors, livers and lungs were sampled for further research.

Hematoxylin and eosin (H\&E) and immunohistochemical (IHC) staining. The histological changes were assessed by H\&E staining (Vector Laboratories, Inc., Burlingame, CA, USA), and the infiltration of neutrophils was assessed by IHC. The tissue samples were fixed in $10 \%$ formalin and embedded in paraffin (Sigma-Aldrich, St. Louis, MO, USA). Paraffin sections were dewaxed with xylene (Merck Millipore, Darmstadt, Germany), rinsed in graded alcohol (Merck Millipore) and rehydrated in water, and subsequently were stained with H\&E for histological examination. The distribution of YB1 in liver tissue was detected by IHC staining. The initial step was the same as H\&E staining, but the paraffin sections then underwent antigen retrieval with citrate buffer (pH 6.0; Abcam, Cambridge, UK). Subsequently, the sections were blocked using 10\% fetal bovine serum (Life Technologies Ltd.; Thermo Fisher Scientific, Inc., Waltham, MA, USA) for $30 \mathrm{~min}$, and rat anti-mouse monoclonal primary antibody (dilution, 1:200; catalog no., 560454; BD Biosciences, Franklin Lakes, NJ, USA) was applied and incubated for $1 \mathrm{~h}$. Following incubation, the sections underwent blocking with 3\% peroxidase (Dako, Glostrup, Denmark) for $30 \mathrm{~min}$, and then goat anti-rat polyclonal secondary antibody (dilution, 1:100; catalog no., 559286; BD Biosciences) was applied and incubated for $30 \mathrm{~min}$. The details of the H\&E and IHC staining processes are described in a previous study by the same group (22).

Cell lines. Human HCC cell lines MHCC-97L and PLC (Japanese Cancer Research Bank; National Institutes of Biomedical Innovation, Health and Nutrition, Osaka, Japan) were maintained in high-glucose Dulbecco's modified Eagle's medium (Life Technologies Ltd.; Thermo Fisher Scientific, Inc.) supplemented with $10 \%$ heat-inactivated fetal bovine serum (Life Technologies Ltd.; Thermo Fisher Scientific, Inc.), 100 mg/ml penicillin $\mathrm{G}$ and $50 \mu \mathrm{g} / \mathrm{ml}$ streptomycin (Life Technologies Ltd., Thermo Fisher Scientific, Inc.) at $37^{\circ} \mathrm{C}$ in a humidified atmosphere containing $5 \% \mathrm{CO}_{2}$. MHCC-97L cells were labeled via stable transfer of the luciferase gene in chromosomes. Briefly, cells were transfected with pGL3 vector (Promega Corporation, Madison, WI, USA), and positive clones were selected according to luciferase activity in the Xenogen IVIS 100 imaging system (PerkinElmer, Inc., Waltham, MA, USA).

Salmonella invasion assay. YB1-incubated MHCC-97L and PLC cells were prepared and co-cultured at a ratio of 1,000:1 for $2 \mathrm{~h}$ under anaerobic $\left(\mathrm{O}_{2}<0.5 \%\right)$ or aerobic conditions. Extracellular bacteria were then removed by washing with phosphate-buffered saline (USB Corporation; Affymetrix, Inc., Santa Clara, CA, USA) and the cells were further cultured with gentamycin medium (Life Technologies Ltd., 
A

\section{Survival proportions after bacteria treatment}
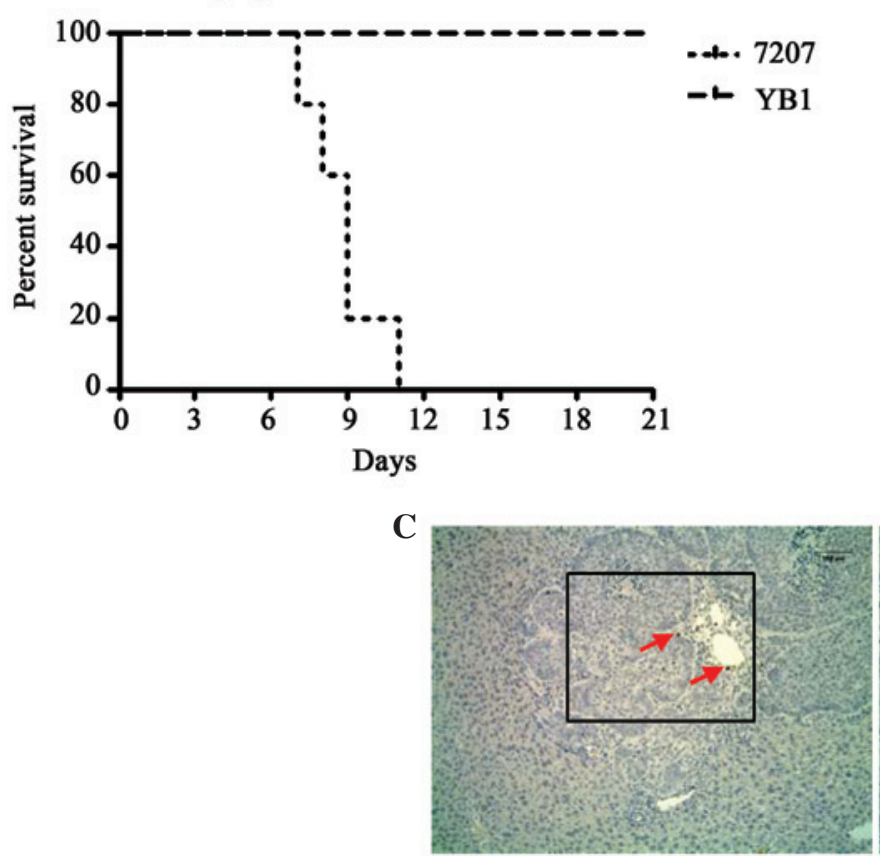

B Percent of body weight change after bacterial treatment

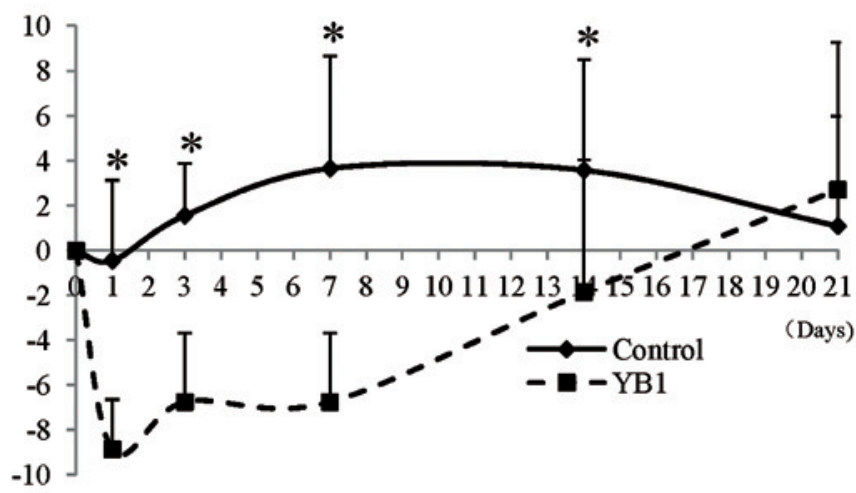

Figure 1. YB1 distribution and effect on nude mice with orthotopic liver tumors (A) Survival rate of nude mice following YB1 and SL7207 injection (n=5 per group). (B) Body weight changes in nude mice were detected on day 1, 3, 7, 14 and 21 following YB1 treatment (treatment, $n=14 ;$ control, $n=9$ ). ${ }^{*} \mathrm{P}<0.05$ compared with mice following YB1 treatment. (C) YB1 was detected on tumor nodules 3 weeks after YB1 treatment (magnification, x100; immunohistochemical staining).

Thermo Fisher Scientific, Inc.) for 24 h. Cells were fixed in paraformaldehyde (4\%) and stained with a rabbit polyclonal anti-Salmonella antibody (dilution, 1:500; catalog no., ab35156; Abcam) for $12 \mathrm{~h}$ at $4^{\circ} \mathrm{C}$. Goat anti-rabbit polyclonal immunoglobulin G Alexa Fluor ${ }^{\circledR}$ 488-conjugated (dilution, 1:100; catalog no., ab150077) and polyclonal horseradish peroxidase-conjugated (dilution, 1:100; catalog no., ab6721) secondary antibodies (Abcam) were added and the cells were incubated for $1 \mathrm{~h}$ at room temperature. Then CytoPainter Phalloidin-iFluor 488 Reagent (dilution, 1:1,000; catalog no., ab176753; Abcam) was applied to indicate cell boundaries. CytoPainter Phalloidin-iFluor 555 Reagent (dilution, 1:1,000; catalog no., ab176756; Abcam) was also applied to demonstrate cell boundaries. The cells were observed under a confocal microscope (LSM 710; Carl Zeiss AG, Oberkochen, Germany).

Detection of cell apoptosis. Cancer cell apoptosis and death induced by YB1 under anaerobic conditions was detected using an Annexin V-propidium iodide (PI) kit (BioVision, Inc., Milpitas, CA, USA) according to the manufacturer's protocol. As demonstrated using flow cytometry (FACSCalibur; (BD Biosciences) Annexin V1/PI- cells were apoptotic and Annexin V1/PI1 cells were dead.

Statistics and data analyses. Continuous variables were expressed as the median with a range. The t-test was used for statistical comparison. The $\chi^{2}$ test was used to compare the incidence of lung metastasis. $\mathrm{P}<0.05$ was considered to indicate a statistically significant difference. Calculations were performed using the SPSS computer software version 16 (SPSS, Inc., Chicago, IL, USA).

\section{Results}

Effect and distribution of YB1 on nude mice bearing orthotopic liver tumors. Orthotopic liver tumor-bearing mice were treated with parental strain SL7207 or engineered strain YB1 for 3 weeks. All mice in the SL7207 group died within 11 days of treatment. All of the mice were alive and healthy 3 weeks after YB1 injection (Fig. 1). To monitor the healthy condition of YB1 treated mice, the body weights were recorded after injection. The results revealed that the body weight decreased by $\sim 9 \%$ on day 1 after YB1 injection and recovered afterwards (Fig. 1B). Notably, we observed that YB1 had low-level distribution in the tumors. This result is different from that observed in our previous study in a breast tumor model (20). On day 21 after treatment, only low amounts of YB1 were identified in the shrunken tumor by paraffin section staining (Fig. 1C). In addition, YB1 was entirely eliminated from the kidney, lymph nodes and spleen 3 weeks subsequent to injection. YB1 was undetected in the blood throughout the experiment.

YB1 significantly suppresses liver tumor growth and metastasis. The effect of YB1 on liver tumor growth and metastasis was studied using an orthotopic liver tumor model in nude mice. Tumor-bearing mice were grouped randomly, and there were no significant differences in the average size of liver tumors between the treatment and control group when the experiment started. Longitudinal monitoring of tumor growth and metastasis was achieved by Xenogen IVIS and the tumor final volume was measured at 3 weeks after treatment. The results indicated that YB1 significantly reduced the size of the primary liver tumor from 1 week to 3 weeks after treatment (Fig. 2A-B). After 3 weeks, the tumors in the control 
Table I. Comparison of tumor size and lung metastasis in mice with or without YB1 treatment.

\begin{tabular}{lccc}
\hline Variable & Control $(\mathrm{n}=9)$ & Treatment $(\mathrm{n}=14)$ & $\mathrm{P}$-value \\
\hline Tumor volume $\left(\mathrm{mm}^{3}\right)^{\mathrm{a}}$ & $580.1 \pm 218.4$ & $61.2 \pm 32.1$ & 0.000 \\
Lung metastasis & $5 / 9(55.6 \%)$ & $0 / 14(0.0 \%)$ & 0.004 \\
\hline
\end{tabular}

aTumor volume, mean \pm standard deviation.
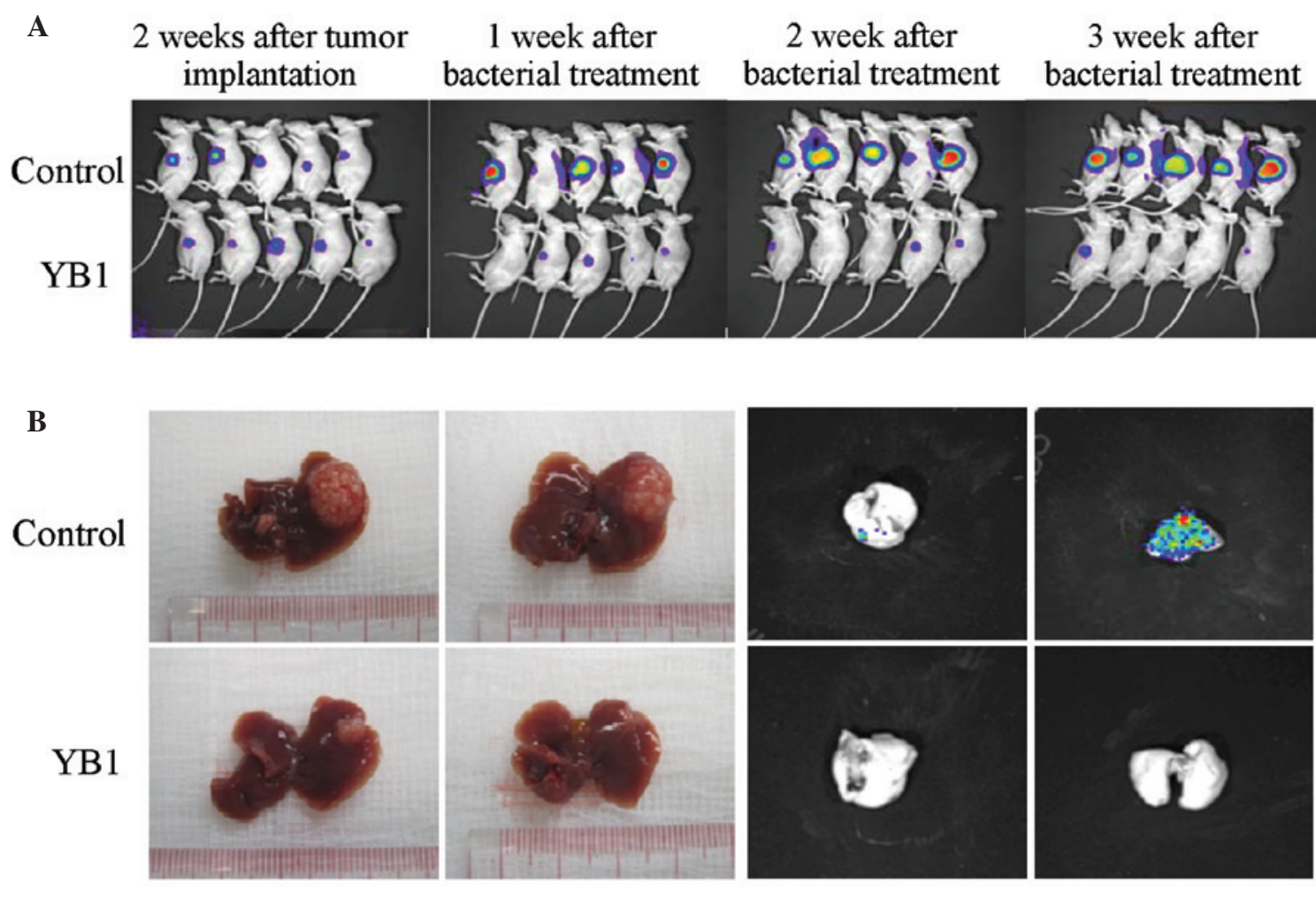

C

Liver

Lung


Figure 2. Comparison of liver tumor growth and metastasis with and without YB1 treatment in a nude mice orthotopic liver tumor model. (A) Tumor growth was monitored by Xenogen IVIS at various time points following YB1 treatment. (B) Liver tumor growth and lung metastasis were inhibited at 3 weeks YB1 treatment. (C) Histological features of liver tumors and lung metastatic tumors 3 weeks after treatment (treatment, $\mathrm{n}=14$; control, $\mathrm{n}=9$ ). Hematoxylin and eosin staining; magnification, $\mathrm{x} 100$.

and YB1-treated groups demonstrated significant differences (580.1 $\mathrm{mm}^{3}$ vs. $61.2 \mathrm{~mm}^{3} ; \mathrm{P}=0.000$; Table I). Lung metastasis was totally repressed by YB1 treatment ( 0 of 14$)$, however, the control group still demonstrated a 55.6\% (5 of 9; $\mathrm{P}=0.004$ ) lung metastasis rate (Table I). These results were additionally confirmed by histological examination (Fig. 2C). 


\section{Control}

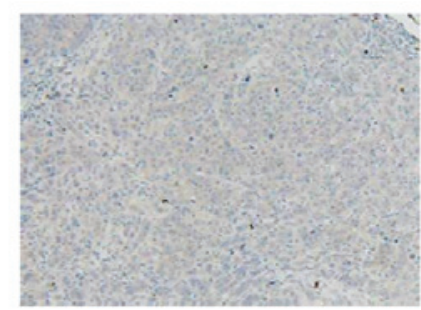

YB1

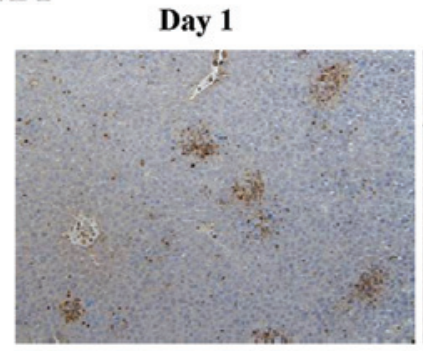

Day 7

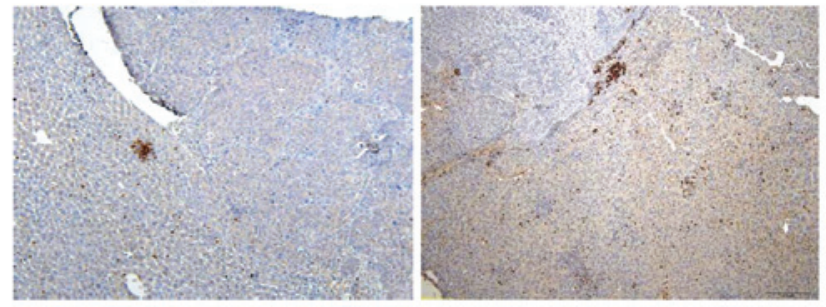

Figure 3. YB1 enhances neutrophil infiltration in liver tissue. Gr1 expression was detected by immunohistochemical staining (treatment, $\mathrm{n}=14$; control, $\mathrm{n}=9$ ). Magnification, $\mathrm{x} 100$.

YB1 enhances neutrophil infiltration in liver tissue. During inflammation of bacterial treatment, circulating neutrophils are recruited to the site of inflammation, which releases a number of various cytokines and enhances the innate immune response (19). In order to investigate the mechanism of YB1 suppression of tumor growth and metastasis, the present study compared the infiltration of neutrophils in the liver between the YB1 treatment and control group. In the control group, only a small number of Grl-positive cells were detected during the entire experiment (Fig. 3). Large amounts of Gr1-positive cells were detected on day 1 to day 21 following YB1 treatment (Fig. 3).

YB1 effectively invades tumor cells and induces tumor cell apoptosis and death. To test the interaction between YB1 and liver cancer, MHCC-97L and PLC cell lines were incubated with YB1 or SL7207 in vitro under anaerobic conditions (oxygen level below 0.5\%). Following the removal of extracellular bacteria and further culturing, it was demonstrated that SL7207 and YB1 effectively invaded the liver cancer cells (Fig. 4A). The effect of SL7207 and YB1 on tumor cells was further investigated by measuring tumor cell apoptosis and death. The results of the present study revealed that an increasing number of dying and apoptotic MHCC-97L cells were detected following co-culture with SL7207 or YB1, relative to the control. The YB1 and SL7207 groups exhibited significant damage to cancer cells under hypoxic conditions
(Fig. 4B). Similar results were observed in the PLC cell line (Fig. 4B).

\section{Discussion}

In the present study, the significant effects of YB1 on the inhibition of HCC growth and metastasis were observed in an orthotopic liver tumor model with distant metastatic potential. With the application of Xenogen IVIS, a notable suppression of liver tumor growth by YB1 treatment was demonstrated longitudinally at different time points. In addition to the inhibition of primary liver tumor growth, YB1 also significantly decreased the incidence of lung metastasis. In contrast with the SL7207 group, all mice in the YB1 group were alive 3 weeks after YB1 injection. Although body weights decreased for a short period, they were restored to their original states within days. The data suggested that YB1 has no severe adverse effects and hence may be a new option in the therapy of HCC patients.

Compared with our previous studies, YB1 treatment in the liver cancer model demonstrated better results, with the primary tumor being greatly repressed and lung metastasis totally eliminated. To explore the underlying mechanism, we investigated two potential directions. First, the innate immune response induced by YB1. A tumor has the ability to escape the immune system due to the development of tolerance. Numerous methods have been used in attempts to alert the immune system to the presence of tumors. In previous studies, immunotherapeutic strategies employ bacteria including Salmonella to enhance the antigenicity of tumor cells (23-25). Studies by Avogadri et al and Al Ramadi et al revealed that attenuated $S$. typhimurium infects malignant cells and triggers an anti-cancer immune response $(26,27)$. Furthermore, $S$. typhimurium plays a significant role in the induction of polymorphonuclear leukocyte migration through its Salmonella invasion protein A (SipA) (28). The present study confirmed that YB1 enhances innate immune response by increasing the infiltration of neutrophils. The results indicate that the suppression effect of YB1 on tumor growth and metastasis may be due to its ability to enhance the innate immune response.

Secondly, due to the ability of YB1 to penetrate deep inside the tumor and gain direct contact with cancer cells, we also further explore the direct effect of YB1 on HCC cells. In the present study, our results revealed that $\mathrm{YB} 1$ effectively invades HCC cells and induces tumor cell apoptosis and death. This is consistent with our previous results (20). A previous study reveals that $S$. typhimurium may activate specific apoptotic enzymes including caspase-3 through $S$. typhimurium effector SipA (29). The activation of caspase-3 in turn increases the infectivity of this pathogen by enhancing the secretion of effectors $(28,29)$. Furthermore, the activation of cleaved caspase- 3 is linked to tumor cell apoptosis (30). Therefore, these data suggested that the suppressive effect of YB1 on tumor growth and metastasis might be attributed to the induction of apoptosis. However, the precise mechanism of YB1 on induced tumor cell apoptosis requires further investigation.

In conclusion, YB1 suppresses liver tumor growth and metastasis by inducing HCC cell apoptosis and enhancing the innate immune response, suggesting that it may be a promising candidate for potential adjuvant therapies for HCC patients. 


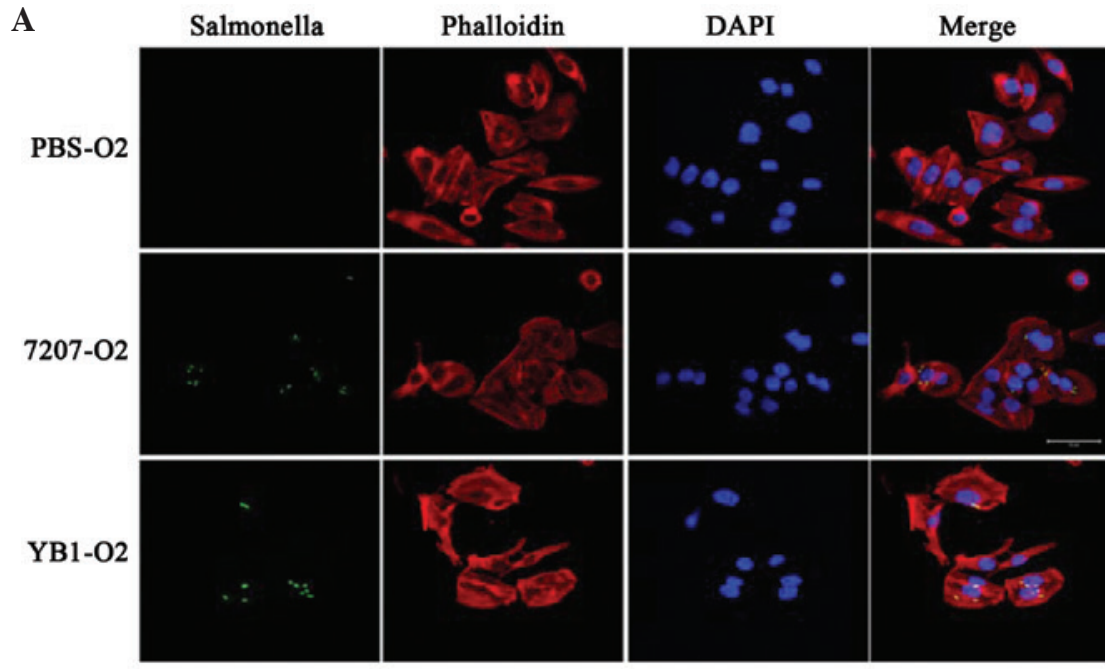

$\mathbf{B}$

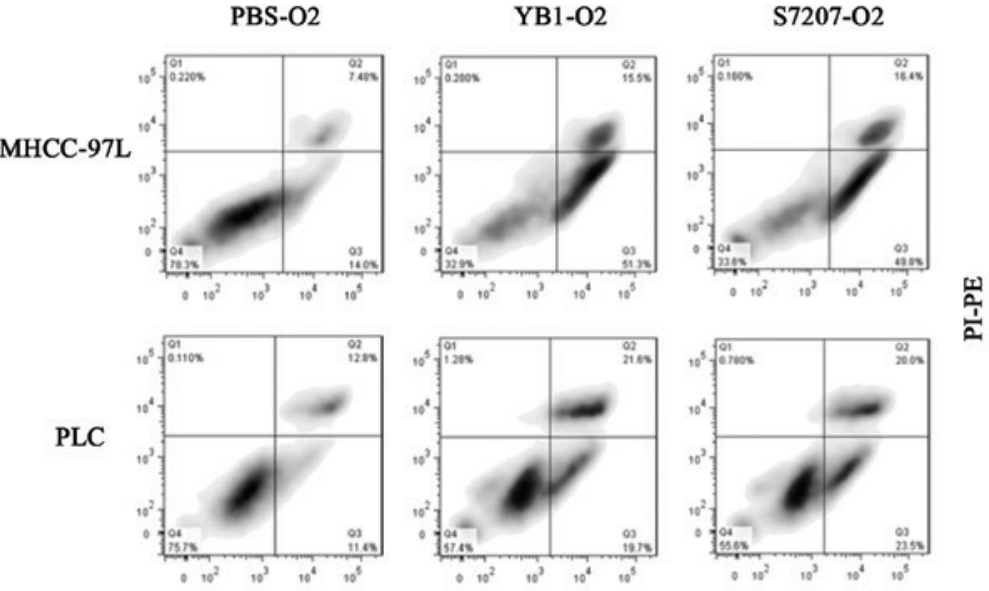

Annexin V-FITC
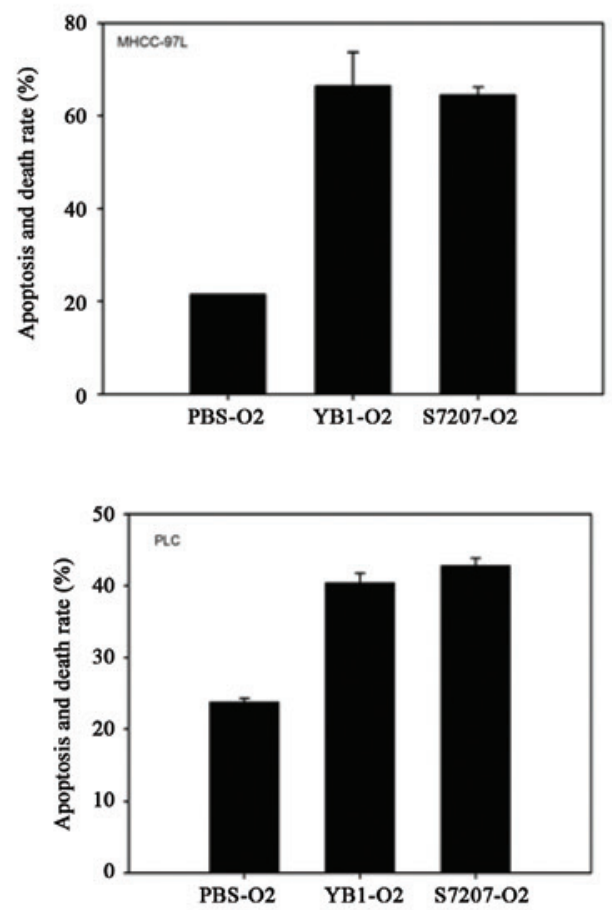

Figure 4. YB1 invades tumor cells and induces tumor cell apoptosis and death. Liver cancer cell lines MHCC-97L and PLC were incubated with YB1 or SL7207 under anaerobic conditions (oxygen level below 0.5\%). (A) YB1 as well as SL7207 effectively invades tumor cells. (B) YB1 induces MHCC-97L and PLC tumor cell apoptosis and death. 


\section{Acknowledgements}

The present study was supported by the Collaborative Research Fund (HKU1/CRF/10 and HKU3/CRF11R) of the Research Grant Council Hong Kong, the National Basic Research Program of China (973 Program, 2014CB745200) from the Ministry of Science and Technology of PRC, the CRCG Seed Funding Program for Applied Research, and the National Science Foundation of China (NSFC) grant no. 31200639.

\section{References}

1. Bosch FX, Ribes J, Díaz M and Cléries R: Primary liver cancer: worldwide incidence and trends. Gastroenterology 127: S5-S16, 2004.

2. Doi K, Horiuchi T, Uchinami M, Tabo T, Kimura N, Yokomachi J, Yoshida M and Tanaka K: Hepatic ischemia-reperfusion promotes liver metastasis of colon cancer. J Surg Res 105: 243-247, 2002.

3. van der Bilt JD, Kranenburg O, Nijkamp MW, Smakman N, Veenendaal LM, Te Velde EA, Voest EE, van Diest PJ and Borel Rinkes IH: Ischemia/reperfusion accelerates the outgrowth of hepatic micrometastases in a highly standardized murine model. Hepatology 42: 165-175, 2005.

4. Bruix J, Llovet JM, Castells A, Montañá X, Brú C, Ayuso MC, Vilana R and Rodés J: Transarterial embolization versus symptomatic treatment in patients with advanced hepatocellular carcinoma: results of a randomized, controlled trial in a single institution. Hepatology 27: 1578-1583, 1998.

5. Ahn J and Flamm SL: Hepatocellular carcinoma. Dis Mon 50: $556-573,2004$

6. Wall DM, Srikanth CV and McCormick BA: Targeting tumors with salmonella Typhimurium - potential for therapy. Oncotarget 1: 721-728, 2010.

7. Pawelek JM, Low KB and Bermudes D: Tumor-targeted Salmonella as a novel anticancer vector. Cancer Res 57: 4537-4544, 1997.

8. Nauts HC, Fowler GA and Bogatko FH: A review of the influence of bacterial infection and of bacterial products (Coley's toxins) on malignant tumors in man; a critical analysis of 30 inoperable cases treated by Coley's mixed toxins, in which diagnosis was confirmed by microscopic examination selected for special study. Acta Med Scand Suppl 276: 1-103, 1953.

9. Barbé S, Van Mellaert L and Anné J: The use of clostridial spores for cancer treatment. J Appl Microbiol 101: 571-578, 2006.

10. Van Mellaert L, Barbé S and Anné J: Clostridium spores as anti-tumour agents. Trends Microbiol 14: 190-196, 2006.

11. Sasaki T, Fujimori M, Hamaji Y, Hama Y, Ito K, Amano J and Taniguchi S: Genetically engineered Bifidobacterium longum for tumor-targeting enzyme-prodrug therapy of autochthonous mammary tumors in rats. Cancer Sci 97: 649-657, 2006.

12. Li X, Fu GF, Fan YR, Liu WH, Liu XJ, Wang JJ and Xu GX: Bifidobacterium adolescentis as a delivery system of endostatin for cancer gene therapy: selective inhibitor of angiogenesis and hypoxic tumor growth. Cancer Gene Ther 10: 105-111, 2003.

13. Patyar S, Joshi R, Byrav DS, Prakash A, Medhi B and Das BK: Bacteria in cancer therapy: a novel experimental strategy. J Biomed Sci 17: 21, 2010.

14. Minton NP: Clostridia in cancer therapy. Nat Rev Microbiol 1: 237-242, 2003

15. Bacon GA, Burrows TW and Yates M: The effects of biochemical mutation on the virulence of bacterium typhosum: the induction and isolation of mutants. Br J Exp Pathol 31: 702-713, 1950.
16. Forbes NS, Munn LL, Fukumura D and Jain RK: Sparse initial entrapment of systemically injected Salmonella typhimurium leads to heterogeneous accumulation within tumors. Cancer Res 63: 5188-5193, 2003.

17. Loessner H, Endmann A, Leschner S, Westphal K, Rohde M, Miloud T, Hämmerling G, Neuhaus K and Weiss S: Remote control of tumour-targeted Salmonella enterica serovar Typhimurium by the use of L-arabinose as inducer of bacterial gene expression in vivo. Cell Microbiol 9: 1529-1537, 2007.

18. Royo JL, Becker PD, Camacho EM, Cebolla A, Link C, Santero E and Guzmán CA: In vivo gene regulation in Salmonella spp. by a salicylate-dependent control circuit. Nat Methods 4: 937-942, 2007.

19. Westphal K, Leschner S, Jablonska J, Loessner H and Weiss S: Containment of tumor-colonizing bacteria by host neutrophils. Cancer Res 68: 2952-2960, 2008.

20. Yu B, Yang M, Shi L, Yao Y, Jiang Q, Li X, Tang LH, Zheng BJ, Yuen KY, Smith DK, et al: Explicit hypoxia targeting with tumor suppression by creating an 'obligate' anaerobic Salmonella Typhimurium strain. Sci Rep 2: 436, 2012.

21. Hoiseth SK and Stocker BA: Aromatic-dependent Salmonella typhimurium are non-virulent and effective as live vaccines. Nature 291: 238-239, 1981.

22. Li CX, Shao Y, Ng KT, Liu XB, Ling CC, Ma YY, Geng W, Fan ST, Lo CM and Man K: FTY720 suppresses liver tumor metastasis by reducing the population of circulating endothelial progenitor cells. PLoS One 7: e32380, 2012.

23. Saccheri F, Pozzi C, Avogadri F, Barozzi S, Faretta M, Fusi P and Rescigno M: Bacteria-induced gap junctions in tumors favor antigen cross-presentation and antitumor immunity. Sci Transl Med 2: 44ra57, 2010.

24. Nishikawa H, Sato E, Briones G, Chen LM, Matsuo M, Nagata Y, Ritter G, Jäger E, Nomura H, Kondo S, et al: In vivo antigen delivery by a Salmonella typhimurium type III secretion system for therapeutic cancer vaccines. J Clin Invest 116: 1946-1954, 2006.

25. al-Ramadi BK, Fernandez-Cabezudo MJ, El-Hasasna H, Al-Salam S, Bashir G and Chouaib S: Potent anti-tumor activity of systemically-administered IL2-expressing Salmonella correlates with decreased angiogenesis and enhanced tumor apoptosis. Clin Immunol 130: 89-97, 2009.

26. Avogadri F, Martinoli C, Petrovska L, Chiodoni C, Transidico P, Bronte V, Longhi R, Colombo MP, Dougan G and Rescigno M: Cancer immunotherapy based on killing of Salmonella-infected tumor cells. Cancer Res 65: 3920-3927, 2005.

27. Al-Ramadi BK, Fernandez-Cabezudo MJ, El-Hasasna H, Al-Salam S, Attoub S, Xu D and Chouaib S: Attenuated bacteria as effectors in cancer immunotherapy. Ann N Y Acad Sci 1138: 351-357, 2008

28. Wall DM, Nadeau WJ, Pazos MA, Shi HN, Galyov EE and McCormick BA: Identification of the Salmonella enterica serotype typhimurium SipA domain responsible for inducing neutrophil recruitment across the intestinal epithelium. Cell Microbiol 9: 2299-2313, 2007.

29. Srikanth CV, Wall DM, Maldonado-Contreras A, Shi HN, Zhou D, Demma Z, Mumy KL and McCormick BA: Salmonella pathogenesis and processing of secreted effectors by caspase-3. Science 330: 390-393, 2010.

30. Ghavami S, Hashemi M, Ande SR, Yeganeh B, Xiao W, Eshraghi M, Bus CJ, Kadkhoda K, Wiechec E, Halayko AJ and Los M: Apoptosis and cancer: mutations within caspase genes. J Med Genet 46: 497-510, 2009. 